\title{
Direct access day case oral surgery
}

\author{
A. Joshi, ${ }^{1}$ L. Doyle, ${ }^{2}$ H. V. Worthington, ${ }^{3}$ and J. P. Rood, ${ }^{4}$
}

Objective To compare the effectiveness of direct access referrals with standard letter referrals, and also assess the impact of the direct access system on the primary care provider, secondary sector and from the patients perspective.

Design Surgical and anaesthetic guidelines were agreed and 12 general dental practitioners were recruited to participate in the study. Each practitioner was given 100 envelopes which contained randomly allocated 50 direct and 50 standard referrals. For direct referrals, GDPs completed a pre-operative assessment and obtained an operation date by telephone from the day case unit while the patient was in the surgery. The clinical history sheet was faxed to the day unit and the radiographs posted. The only contact the patient had with the hospital was on the day of surgery. Standard referral patients were referred in the traditional way with a referral letter being sent to an out-patient clinic. Evaluation of the effectiveness of the direct referral system versus standard letter method were made via completion of questionnaires by the patients, dental practitioners, hospital clinicians, day case anaesthetist and co-ordinator.

Setting The study was carried out over 2 years commencing in 1997 at the Oral Surgery Day Case Unit at Manchester Royal Infirmary.

Results A greater number of direct access referrals (409; 90\%) were treated in comparison with $(312 ; 75 \%)$ standard referrals $(P<0.001)$. Eighty nine per cent of direct access records were adequate pre-operatively. More than $70 \%$ of dentists and hospital clinicians preferred the direct access referral method. Sixty-three per cent of standard letter patients were satisfied with their mode of referral compared with $87 \%$ of direct access patients.

Conclusions Given appropriate guidelines dental practitioners are able to refer directly to oral surgery day case operation lists.

This has proved to be the favoured method of referral for dental practitioners, the secondary sector and patients.

$\mathrm{C}^{\text {or routine dento-alveolar surgery, general dental practitioners }}$ F (GDPs) normally send a referral letter to a hospital consultant (standard letter referral). The letter is then forwarded to the reception staff for an out-patient consultation. The patient's first visit is for consultation, and if day surgery is advised then a second visit is made for a pre-admission clinic, where the patient receives an operation date and has a third visit to hospital for surgery. Some patients may require a review appointment. The patient therefore has three visits to hospital for routine oral surgical procedures.

When a GDP refers a fit and healthy patient with a defined diag-

$1^{*}$ Lecturer (Honorary Senior Registrar) in Oral \& Maxillofacial Surgery

${ }^{2}$ Consultant Anaesthetist, Manchester Royal Infirmary, Oxford Road, Manchester M13 9WL ${ }^{3}$ Reader in Dental Statistics, Department of Dental Medicine and

Surgery, University Dental Hospital of Manchester M15 $6 \mathrm{FH}^{4}$ Consultant in Oral $\ll$ Maxillofacial Surgery, GKT School of Dentistry, London SE5 9RW

${ }^{*}$ Correspondence to: Dr A Joshi, Lecturer/Honorary Senior Registrar, Department of Oral and Maxillofacial Surgery, Manchester Royal Infirmary, Oxford Road, Manchester M13 9WL

REFEREED PAPER

Received 14.09.99; accepted 7.12.99

() British Dental Journal 2000; 188: 452-456 nosis for routine dento-alveolar surgery, the direct access (DA) system for day surgery can be recommended, whereby the patient only attends hospital on one occasion for surgery.

GDPs are highly trained and experienced and are familiar with patients under their care. While there is a range of competence among GDPs, most are able to diagnose dento-alveolar procedures requiring day surgery. Although GDPs may not have formal training in pre-operative assessment, they have knowledge of the patient's past medical, dental and family history, and provided they follow clear guidelines, they should be able to refer patients directly to a day surgery operating session.

'Fastrak' services have been reported for general surgery in South Tyneside and the authors stated that if each general medical practitioner referred eight patients per year the savings on out-patients appointments would be equivalent of one new patient clinic per week. ${ }^{1}$ Direct listing for adult tonsillectomy has been estimated to save the NHS 2 million pounds per annum. ${ }^{2}$ Recently, Renton et al. examined direct listing as a realistic proposition for oral surgery and reported that a one visit day case system proved effective and feasible to provide. ${ }^{3}$ GDPs are capable of selecting patients for day case oral surgery on both surgical and anaesthetic grounds. ${ }^{4}$ The selection criteria for fitness of a patient for inclusion into the direct access study (Figure 2) were derived from the Royal College of Surgeons' Guidelines ${ }^{5}$ and recommendations from the Audit Commission. 6,7 The criteria used for selection of surgical procedures suitable for day case oral surgery (Figure 3 ) were based on current clinical practice and recommendations in the medical literature. ${ }^{8-10}$ If the proposed one visit system (whereby the patient is assessed, operated on and discharged on the same day) proved effective it would encourage the move from inpatient to day case surgery and, apart from reducing cost, would reduce the number of hospital visits for the patient.

This study aims to compare the effectiveness of direct access (DA) referrals with standard letter referrals (SR), and also assesses the impact of this service on the primary care providers, the secondary sector and from the patients' perspectives.

\section{Method}

This collaborative research study between hospital clinicians and dental practitioners was undertaken in the adult Oral Surgery Day Case Unit at Manchester Royal Infirmary (January 1997 - April 1999). GDPs in the Manchester area were invited to participate in the study and protocols were discussed and agreed in meetings. Twelve GDPs were randomly recruited to the study from the Manchester area. Each GDP was provided with 100 envelopes which contained 50 direct referrals and 50 standard referrals in a random order which was double blind to the investigators and practitioners. Figure 1 outlines the route involved with each method of referral. Each practice was also provided with study literature, Hemocue machine (for haemoglobin assessment), weighing scales, BP monitor and fax machine. A research co-ordinator was employed to arrange admissions for the direct referral patients. For patients of Afro-Caribbean and Mediterranean origin, further haematological investigations to establish the sickle status were obtained by means of Sickle Dex tests. The test was arranged at the request of the GDPs 
Experienced GDP refers fit individual with a clear, certain diagnosis

Treatment discussed
Direct Referral

Referral letter sent to oral surgery day case unit $\downarrow$

Out-patient appointment sent to patient

Patient attends out-patient clinic

$\downarrow$ Hospital clinician finalises treatment plan, repeats radiographs

Date given for operation 2-4 weeks later $\downarrow$

Patient has operation, discharged same day May be reviewed 14 days later

*OSDU is the oral surgery day case unit

Figure 2 Selection criteria for fitness of patients for inclusion into the direct access study

- Patients must be less than 60 or more than 16 years of age

- Be less than $100 \mathrm{~kg}$ in weight

- Be ambulant and have no systemic illness which affects their lifestyle

- If haemoglobin estimation using the hemocue machine is less than $10 \mathrm{~g} / \mathrm{dl}$ and systolic BP > 160: diastolic BP > 100, patient does not meet selection criteria

- Be accompanied home by an escort in a car or taxi, and then be cared for at home for the first 24 hours

- Patient must agree not to drive or use machinery for 24 hours post-operatively

\section{Figure 3 Day case surgical procedures}

Surgical procedures to be performed under general anaesthesia or local anaesthesia supplemented with intravenous sedation:

- Extractions < 12 teeth/roots

- Removal of symptomatic third molar teeth

- Removal of impacted teeth for orthodontic purposes

- Apical surgery

- Soft tissue and pre-prosthetic surgery

- Enucleation of cysts

by the research co-ordinator, and a small number of patients attended the hospital for the relevant blood test pre-operatively.

When a patient required routine dento-alveolar surgery under general anaesthesia or intravenous sedation, and fulfilled the day case anaesthetic and surgical criteria, (Figures 2 and 3 ), the practitioner opened an envelope to establish which method of referral was to be employed for that particular patient. If the patient was to be a standard letter referral, the practitioner followed the traditional referral method and the patient attended for consultation. GDPs were also aware that they could refer patients for out-patient consultation if a specialist opinion was required prior to surgery. For a direct access referral, the patient's pre-operative assessment was completed by the practitioner and her or his dental nurse, and an operation date was obtained from the research co-ordinator whilst the patient was still in the surgery. The dental practice then faxed a clinical history sheet to the co-ordinator so that clinical notes were made available prior to the patient's arrival for surgery. The GDPs were also provided with self-addressed second class stamped envelopes to forward relevant radiographs prior to the patient's surgery. The only contact the day unit had with the patient was on the day of surgery. A discharge letter was faxed post-operatively to the GDPs by the research co-ordinator on the same day.
Figure 4 GDP/hospital clinician questionnaire

GDP ....... Hospital Clinician .......

1. Was the patient willing to be a direct referral/referral by standard letter?

If no, why? Yes No

2. Was the patient's history sheet comprehensive and easy to complete? Yes No

If no, give details so that consideration can be given to modify the form.

3. How long did it take to complete the patient history sheet and make arrangements for your patient's admission? ...........mins

4. Did you/your dental nurse experience any difficulties in contacting our Day Surgery Unit Coordinator?

Yes

No

If yes, in what way?

5. Did your patient receive an appointment for their operation at a convenient date?

If no, why?

Yes No

6. Did you receive a discharge summary and other correspondence from our unit in time for your patient to be reviewed in your surgery? Yes No

If no, did you contact and receive the information from the day surgery Coordinator?

7. How many times did your patient consult you after their operation? a) One visit for planned review

b) More than one visit

c) As an emergency

Why?

8. Did you refer the patient back to the hospital after discharge?

If yes, where

Yes

No

a) A\&E Department

b) Out-patient Consultant Clinic

c) Other

Reason for Referral:

9. What is your preferred method of referral for this patient?

a) Direct Referral

b) Referral by standard letter,

c) Either

(please tick (a), (b) or (c)

10. What are the advantages/disadvantages of processing this patient as a direct referral as opposed to referral by standard letter? 
All faxes were dealt with immediately during working hours by the research co-ordinator and out of hours the room was locked, thereby maintaining the security of confidential patient information.

For the purposes of this study, patients referred via the direct access method were reviewed by their GDP 2 weeks after surgery. This appointment was made in order to assess if the GDPs had to deal with any complications from the direct access patients postoperatively. Standard letter patients were not routinely reviewed post-operatively.

There were five general anaesthetic day case operating lists and ten intravenous sedation sessions available for the study, of which only one of each list was dedicated to receive direct access patients. A comparison of the effectiveness of the two methods was made by completion of questionnaires by the surgeons, anaesthetist, hospital clinicians, GDPs, patients and research coordinator (Figure 4 shows an example of the questionnaires completed by the GDP/hospital clinician and Figure 5 is the patient questionnaire).

\section{Figure 5 Patient questionnaire}

I would be most grateful if you could complete the short questionnaire below. The information you provide will help with ongoing research within our unit and be treated in the strictest confidence.

1. Were you referred directly for your operation by your dentist? Yes No

2. How many visits have you had to our hospital regarding your operation today?

a) none/came directly from dentist b) 1 c) 2 d) other

3. If you have attended a pre-admission clinic, would you be happy for your dentist to refer you directly for your operation?

$$
\text { Yes No No Preference }
$$

4. Did you feel there were any disadvantages to attending our clinic? If so, please give details.

5. Did you feel there were any advantages to attending our clinic ? If so, please give details.

6. How long have you waited between your clinic appointment and treatment today? $\quad$...........weeks.

7. After your operation today, would you be happy to be reviewed by your dentist rather than attend our clinic at the hospital.

$$
\text { Yes }
$$

Please give your reasons why?

\section{Figure 6}

Advantages of processing patients as direct referrals as opposed to standard referrals (GDP comments)

- Direct access quicker and easier as opposed to standard letter process being lengthy and time consuming.

- Less appointments for patients to attend

- Operation date given immediately and GDP aware of patient's progress

- Shorter waiting times for surgery

- Ideal for patients who need to be seen urgently

- Less confusion and trauma for the patient

- Made convenient for patient's family and work commitments

Disadvantages of processing patients as direct referrals as opposed to standard letter referrals (GDP comments)

- No fee for direct access patients

- History sheet sometimes time-consuming

- Standard letter may be quicker to process during surgery hours

- Some patients need consultation in hospital prior to surgery
Table 1 Reasons why direct access (DA) and standard letter (SR) patients did not proceed with surgery

\begin{tabular}{lcr} 
Reasons for failure to proceed to surgery & Number of patients \\
& DA & SR \\
\hline & & \\
DNA consultation & 11 & 22 \\
DNA surgery & 18 & 12 \\
Patient cancelled consultation & 3 & 17 \\
Patient cancelled surgery & 6 & 1 \\
Treatment not necessary & 7 & 3 \\
Patient refused treatment & & \\
Medically unfit & & \\
\hline
\end{tabular}

Chi-squared tests were used to compare different factors between direct access and standard letter referrals. A significance level of 0.05 was used throughout.

\section{Results}

From the 1,200 study envelopes distributed between the 12 GDPs, 872 referrals were received of which 454 were direct access referrals, and 418 were standard letters. Three hundred and twenty eight referral envelopes were returned unopened. A greater number of direct access referrals $(409 ; 90 \%)$ were treated in comparison with 312 (75\%) standard referrals $(P<0.001)$. Nearly two thirds of the patients had third molar surgery $(517 ; 72 \%), 16 \%$ (114) patients had surgical removal of teeth, and $12 \%(84)$ had apical surgery.

The reasons why patients did not proceed to surgery are shown in Table 1. Forty-two (10\%) of the standard referrals failed to attend for consultation. In the direct referral group, $11(2 \%)$ patients did not attend for surgery; however a significantly greater number of patients $(22,5.3 \%)$ failed in the standard letter group $(P<0.001)$. A significantly greater number of patients $(18,4 \%)$ in the direct referral group cancelled their operation compared with standard referral patients $(12,2.9 \%)$.

A number of patients were not treated as they did not fulfil the surgical and anaesthetic criteria. Ten patients (7 DA, $3 \mathrm{SR}$ ) were medically unfit on the day of operation; three patients in the standard referral group were underage; nine patients had treatment performed privately following referral ( $3 \mathrm{DA}, 6 \mathrm{SR})$ and seven patients refused treatment (6 DA, $1 \mathrm{SR})$. There was a difference between the two groups in the number of patients for whom treatment was not deemed necessary $(P<0.001)$. Direct access patients had access to operating lists within a $2-3$ weeks whereas standard letter patients waited from 2-28 weeks for an operation date as some patients were subject to contractual restrictions.

From the questionnaires, the following series of evaluations were made:

\section{The ability of general dental practitioners to refer} appropriately

a) Anaesthetic assessment. A minority of patients were cancelled on the day of surgery by the anaesthetist, because of complications in the anaesthetic room. One patient showed a bigemenic rhythm on ECG and two patients suffered bronchospasm on induction of general anaesthesia and therefore their operations were abandoned. All but three patients referred for surgery under general anaesthesia were ASA1. The carer was available for $98 \%$ of direct referrals and $99 \%$ of standard referral patients.

b) Number of appropriate cases. For a significant number of standard referrals $(77 ; 23 \%)$, treatment was not performed as per the original request by the GDP compared with $(31 ; 8 \%)$ in the direct access group $(P<0.001)$. The reasons for this include: 10 patients $(7$ DA; 3 SR) were unfit for operation on the day of surgery but were appropriately referred; treatment was not necessary in 20 patients (3 DA; 17 SR); 17 patients (5 DA; 12 SR) had treatment performed under local anaesthesia only; 3 patients (1DA; 2SR) had an incorrect 
diagnosis and 33 patients (10 DA, $23 \mathrm{SR}$ ) had only symptomatic lower third molar teeth removed instead of all four third molar teeth; 20 patients (5 DA; 15 SR) had treatment performed under local anaesthesia with intravenous sedation instead of a general anaesthesia and 5 standard referral patients deferred treatment.

c) Adequate preoperative records. Eighty nine per cent of DA records were adequate pre-operatively compared with more than $99 \%$ of SR clinical notes $(P<0.001)$. The main reason for pre-operative records being inadequate was that the radiographs forwarded by GDPs did not arrive in time for surgery or were inadequate. Two patients reported a clinical history other than that recorded by the GDP. The haemoglobin or sickle status was not available for three patients and the mode of analgesia to be used for surgery for one patient was not stated.

d) Adequate patient information pre-operatively. Ninety five per cent of direct referral patients had good knowledge of their procedure pre-operatively compared with $99 \%$ standard letter patients $(P<0.001)$. For $28(5 \%)$ of the direct access patients an explanation of the surgical procedure was given but patients recall was vague. Thirty-eight $(9 \%)$ direct access patients were not aware of the morbidity associated with their operation compared with 3 $(1 \%)$ of standard letter patients $(P<0.001)$.

e) Number of operations cancelled by surgeon. There were no statistically significant differences in the number of operations cancelled in each group (8 DA, $3 \mathrm{SR}, P=0.28$ ). Four direct access referrals were not fit to have their operation on the day of surgery because three patients had complications in the anaesthetic room and one patient was not starved. Two patients were asymptomatic and wished to delay their operation, and another patient referred for surgery under general anaesthesia was not aware that he required a minimum of 24 hours for recovery post-operatively. One patient was not sure whether he wished to proceed with surgery on the day, therefore his treatment was deferred. Three standard letter patients attended on the day of surgery with upper respiratory tract infections and surgery was deferred.

\section{Ease of collation of pre-operative information for co-ordinator}

The co-ordinator had complete pre-operative records for $95 \%$ of direct referrals. Twenty-one patients did not have radiographs available or their arrival was delayed in the post. Three patients did not attend for a Sickle Dex test.

\section{Professional satisfaction with the two referral methods}

a) Time taken to arrange admission for direct access patients. All but one of the GDPs found the clinical history sheet easy to complete compared with $99 \%$ of hospital clinicians. There was a statistically significant difference between the time it took hospital clinicians to complete the history sheet and arrange patient's admission (mean of $15, \mathrm{SD}=5$ minutes) compared with GDPs who took 20 minutes $(\mathrm{SD}=9)(P<0.001)$.

b) Preferred method of referral. Seventy seven per cent of hospital clinicians stated that they preferred the direct access referral method in preference to standard letters. GDPs showed a similar preference (74\%). The advantages the GDPs and clinicians stated for this mode of referral are outlined in Figure 6. The GDPs experienced difficulty in contacting the day unit co-ordinator for 23 direct referral patients because her or his working day finished at $4.00 \mathrm{pm}$.

\section{Patient satisfaction}

Direct access patients showed an $87 \%$ satisfaction rate with their mode of referral compared with $63 \%$ of standard letter patients. Fifty patients did not state a preference for either method of referral. All direct access patients had one visit compared with $91 \%$ of standard letter patients who had two visits and $9 \%$ who had three visits to hospital. Standard letter patients outlined the following disad- vantages to attending hospital for consultation: parking problems; waiting time for a consultation was too long; and, difficulty in finding the department. The advantages of attending the out-patient clinic were: meeting the staff responsible for the treatment; obtaining a second opinion from a hospital clinician and having the opportunity to ask questions. Seventy per cent of standard letter patients preferred to be reviewed post-operatively by their GDP as it was more convenient to attend their local dental practice.

\section{Discussion}

Day surgery is recognised as an area for expansion and development by the Royal College of Surgeons, and the Audit Commission. 5,11 The main outcome measures in this study were satisfaction of the primary sector, secondary provider and patients with the direct and standard letter method of referral.

Seventy four per cent of GDPs preferred the direct access referral route. GDPs needed $20(\mathrm{SD}=9)$ minutes to complete the history sheet, arrange admission and were satisfied with the pre-operative completion of records. This presented an additional demand on the practitioners' clinical time for which they were not remunerated. A total of 328 referral envelopes were unused. At an early stage of the study, one GDP left his practice and two other referring GDPs offered surgical dentistry under sedation in their practices and subsequently did not refer many patients. However the remaining nine practitioners were enthusiastic. The direct referral method should work optimally when used by enthusiastic practitioners who pay careful attention to selection criteria. ${ }^{3}$ Unlike the report of Renton et al., ${ }^{3}$ treatment plans were altered on the day of surgery for direct access patients. Some patients had surgery under intravenous sedation instead of general anaesthesia, and treatment plans were altered following discussions with patients who were keen to have treatment under local anaesthesia without intravenous sedation. It is difficult to determine precisely whether these patients were assessed inappropriately by practitioners or whether the patients changed their mind on the day of surgery. All patients received surgery on a day case basis and were not re-directed for inpatient surgery, except for three patients who had complications following induction of anaesthesia. On the whole, patients were therefore selected appropriately for direct access surgery by a small group of enthusiastic GDPs.

Seventy seven per cent of hospital clinicians preferred the direct referral method because patients' admission could be arranged to suit their personal and work commitments. Standard out-patient referral by letter does allow assessment by a consultant or other member of staff backed by the full resources of the X-ray Department. However, there may be delays inherent in postage and in administration. A dedicated facsimile-based system for semi-acute referrals has been successfully used in ophthalmology 12 method adopted in this study. The running costs of this method of communication were minimal.

The co-ordinator had minimal difficulties in collating patient information. Some of the radiographs did not arrive in time for surgery because of postal delays. Ten patients radiographs were of poor quality and required repeating prior to surgery.

The success of direct access referrals is measured by the number of patients who had surgery on the day of attendance; $98 \%$ of direct referral patients proceeded to surgery. The study design was limited in that it only offered two options for surgery either under local anaesthetic with intravenous sedation or general anaesthesia. Some practitioners may have unnecessarily selected one of these methods rather than treatment under local anaesthesia to ensure that their patients were accepted into the study; a small proportion of patients 17 (5 DA; $12 \mathrm{SR}$ ) were treated under local anaesthesia. The other main reason why treatment plans were altered were that only symptomatic third molar teeth were removed in line with current guidelines. 
Patients in the direct referral group were very satisfied with their referral method and only $4 \%$ cancelled their operation. They were impressed by the lack of delay in obtaining a date for surgery that suited their personal and professional commitments. This is in contrast to a previous report (Renton et al. ${ }^{3}$ )where the ability to provide surgery within a short period of referral did not appeal to most patients. A significantly greater number of standard letter patients did not attend for consultation and surgery. A small number of direct referral patients also failed to attend for surgery. Failure of patients on the day of surgery may result in wasted resources in the day case operating facilities. The research co-ordinator contacted most of the standard letter patients who failed to attend for consultation. The main reasons for non-attendance was either inconvenient date and time of appointment or that an outpatient appointment was 'never received'. The wasted resources in out-patient clinics with a significant failure rate for consultations must not be under-estimated.

\section{Conclusion}

Direct access referral is acceptable to GDPs, the secondary sector and to patients. It shifts costs and responsibility to the primary sector with obvious savings for the secondary provider. With appropriate clinical and anaesthetic guidelines, GDPs are able to refer appropriately for direct access surgery. However, this study involved a very small sample of GDPs who may not be representative of practitioners as a whole. The recruited dentists were enthusiastic and were willing to participate in research. It could be argued that if the direct referral method was in wider use outside such research the results may not be as favourable. Furthermore, GDPs were not financially rewarded for the additional responsibility placed on them.
The authors are grateful to Miss B Hanif, Research Co-ordinator for her help with the study. We would also like to thank all the general dental practitioners who participated in this study. This work was supported by a grant from the North West NHS Reactive Funding Scheme.

1 Bradshaw C, Pritchett C, Eccles M, Armitage T, Wright H, Todd E. South Tyneside 'Fastrak' day surgery planning. J Day Surg 1994; 3: 6-8.

2 Shah C, Griffiths M, Moralee S Direct listing for adult tonsillectomy. JR Coll Surg Edinb 1997; 42: 45-46.

3 Renton T, McGurk M. Direct referral day case oral surgery for dental practitioners: a pilot investigation. Br Dent J 1999; 186: 334-337.

4 Joyson O B, Williams S L, Brickley M R, Shepherd J P. Lower third molar treatment planning ability of general dental practitioners and oral maxillofacial surgeon using receiver operating characteristics methodology. Br Dent J 1996; 181: 411-415.

5 Guidelines for day case surgery. London: Roy Coll Surg Eng 1985, revised March 1992.

6 Measuring the quality: the patient's view of day surgery. Audit Commission, NHS occasional papers, No. 3, May 1991.

7 The Audit Commission for local authorities and the National Health Service for England and Wales. A short cut to better service. Day surgery in England and Wales. London: HMSO, 1990.

8 Mercier P, Precious D. Risks and benefits of removal of impacted third molars: a critical review of the literature. Int J Oral Maxfac Surg 1992; 21: 17-27.

9 Shepherd J. Evidence based indications for third molar removal. Dent Defence Union Focus 1996; 2: 8-10.

10 Weil T M, Akerson H A, Alling C C, Davis Jr W M. Parameters of care for oral and maxillofacial surgery: A guide for practice, monitoring and evaluation. (AAOMS parameters of care in dento-alveolar surgery). J Oral Maxfac Surg 1995 (supplement 5); 53: 31-59.

11 Audit Commission. All in a day's work - and audit of day surgery in England and Wales. London: HMSO, 1992.

12 Dayan M, Fitt A, Bosanquet R. A facsimile — based system for semi-acute hospital referrals - the experience in ophthalmology. Health Trends 1996; 28: 20-21. 